\title{
The Applications of Mathematical Modeling Based on MATLAB
}

\author{
MingE SHA ${ }^{1}$, Yonggang XIE ${ }^{2}$ \\ ${ }^{1}$ Department of Mathematics, Kunming University, Kunming, 650214, CHINA \\ ${ }^{2}$ Equipment Management Department of Kunming University, Kunming, 650214, CHINA \\ ${ }^{1}$ MingE_Sha@126.com, ${ }^{2}$ ygxie@kmu.edu.cn
}

\begin{abstract}
Mathematical Modeling can be widely used in the graph displaying and solve many mathematical problems in an easy, visual and even dynamic way. Using Matlab software in the teaching of mathematics and other subjects makes teaching and learning interesting and easier. This paper presents the concept, method and steps of mathematical modeling and gives some useful examples. Using Matlab in Mathematical teaching can improve teaching quality and improve students' academic performance, which plays an important role in Mathematical modeling and mathematic teaching.

Index Terms -matlab, modeling, rayleigh fading, distribution, probability density function (pdf)
\end{abstract}

\section{Introduction}

Due to interesting, audio-visual, figurative, flexible advantages, mathematical modeling becomes a great tool for most teachers teaching their lessons, especially those who teach mathematics. However, some teachers may be reluctant to include modeling activities into classroom time, because modeling takes significant time and effort to adjust parameters and make programs to run properly. Fortunately, MATLAB provides high flexibility for teachers to solve programming problems easily.

MATLAB is a high-level language tool for numerical computation and visualization. It works in a multiparadigam numerical computing environment and allows matrix manipulations, plotting of functions and data, implementation of algorithms, creation of user interfaces, and interfacing with programs written in other languages, including C, C++, Java, Fortran and Python[1].

Mathematical modeling is the process that we observe the various phenomena and behaviours of real world, import some mathematical symbols, variables and parameters, and use mathematical language and method to reflect the inner relationships of these variables and parameters, and produce a mathematical model eventually. Mathematical model is a representation in mathematical terms of the behaviour of real devices and objects. The process of mathematical modelling has seven stages: preparation, observation, prediction, modelling, analysis, detection and application. Normally, this process requires dealing with large sets of data which impossible to handle by hand-calculation. MATLAB has powerful data processing ability, which can solve problems in a very short time period, thus allowing the speed of innovation cycles to be increased. This ensures a potential advantage to industries, which can save time and money in the development and validation phases.
In this paper, we study some applications of mathematical modeling and show the advantages of MATLAB in mathematical teaching. The remainder of this paper is organized as follows: the concept of mathematical modeling and modeling steps are presented in Section II. In Section III, two basic applications in Mathematics based on Matlab are studied. A car number prediction model is analyzed and simulated in Section IV. Section V Simulation studied the Rayleigh distribution, and section VI concludes the paper.

\section{Mathematical Modeling}

Mathematical model is a representation in mathematical terms of the behavior of real devices and objects. It describes situations in everyday life using formulas, which enable us to better understand the situations. Mathematical modeling is a principled activity. The methodological modeling principles are as following [2] [3]:

- Study the origin problem in the real world and simplify it to a real model;

- Understand the needs for the model;

- Select basic elements, simplify and define them in a precise way;

- List the data we are seeking and indentify the available relevant data;

- Identify the circumstances and identify the governing physical principles;

- Use mathematical symbols, relations and mathematical operations to express the problem and obtain the resulting mathematical system;

- Study the resulting mathematical system using appropriate mathematical ideas and techniques;

- Compare the results predicted on the basis of the mathematical work with the real world;

- Identify tests that can be made to validate and verify the model.

- Implement the "model-validate-verify-improve-predict" loop. 


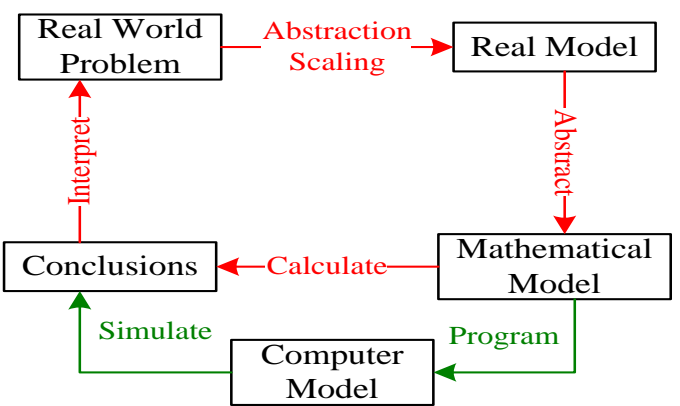

Fig. 1 Mathematical Model Building

The looping, iterative structure of mathematical model building shows in Fig.1.

\section{Basic Applications in Mathematics}

Using Matlab, we can do many things more than we could think about. For example, plotting function graphs which make its easer for students to better understand many tedious mathematical formulas.

Example. 1. Visual studying the function $z=f(x, y)$

$$
z=\frac{\sin \sqrt{x^{2}+y^{2}}}{x^{2}+y^{2}},-7.5 \leq x \leq 7.5,-7.5 \leq y \leq 7.5
$$

Matlab solution

clc; $\quad \%$ clear the command window

clear all; $\%$ clear all the variables

close all; $\%$ close all figure windows

$\mathrm{x}=-7.5: 0.5: 7.5 ; \%$ define variable $\mathrm{x}$

$\mathrm{y}=\mathrm{x}$;

$[\mathrm{X}, \mathrm{Y}]=\operatorname{meshgrid}(\mathrm{x}, \mathrm{y}) ; \quad \%$ define $\mathrm{X}, \mathrm{Y}$ arrays

$\mathrm{R}=\operatorname{sqrt}\left(\mathrm{X} \cdot{ }^{\wedge} 2+\mathrm{Y} .{ }^{\wedge} 2\right)+\mathrm{eps} ; \%$ add eps in case of $0 / 0$ $\mathrm{Z}=\sin (\mathrm{R}) . / \mathrm{R}$

Mesh (X, Y, Z) \% three-dimensional gridding

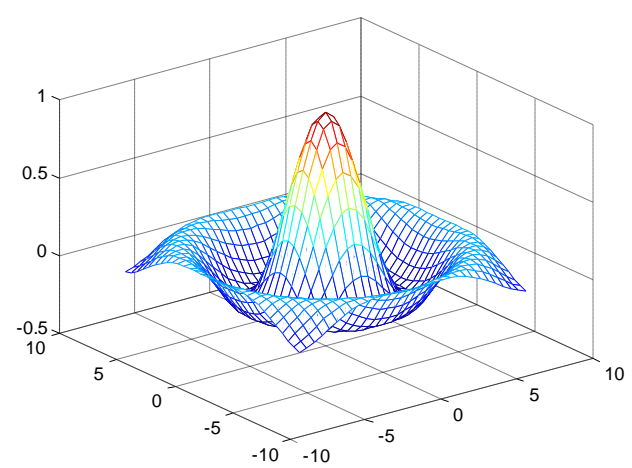

Fig. 2 The mesh plot of sinc function

Fig. 2 is the mesh plot of sinc function, and if use the "surf" to take the place of the "mesh", we create colored parametric surfaces specified by X, Y, and Z, see Fig. 3 .

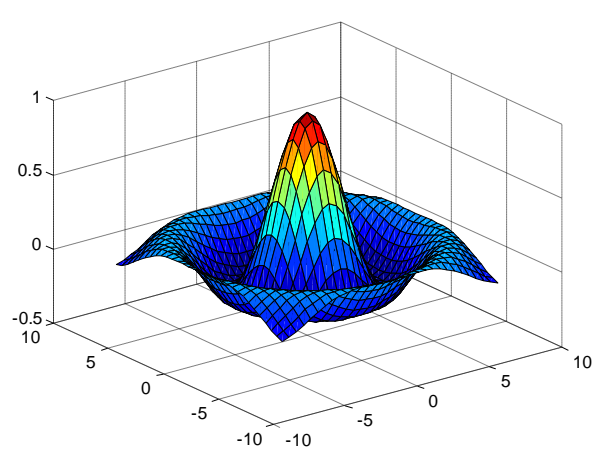

Fig. 3 The surface plot of sinc function

Another example is that we can use stem 3 to visualize the function $y=4-8 * e^{-s t / 3}$ at different angles of view.

Matlab solution

$\mathrm{t}=0: 0.15: 35$;

$\mathrm{s}=0.1+\mathrm{i}$

$\mathrm{y}=4-8 * \exp (-\mathrm{s} * \mathrm{t} / 3)$;

stem3 (real(y), imag $(y), t)$

hold on

plot3 (real(y), imag (y), t, 'r')

hold off

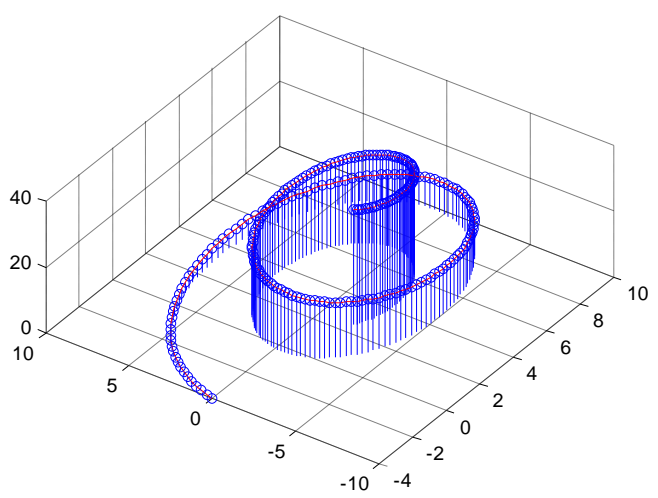

Fig. 4(a) different view of the stem plot $y=4-8 * \exp (-s t / 3)$

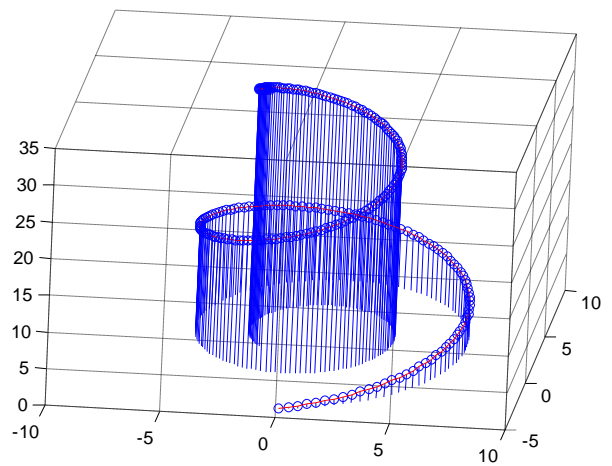

Fig. 4(b) different view of the stem plot $y=4-8 * \exp (-\mathrm{st} / 3)$ 


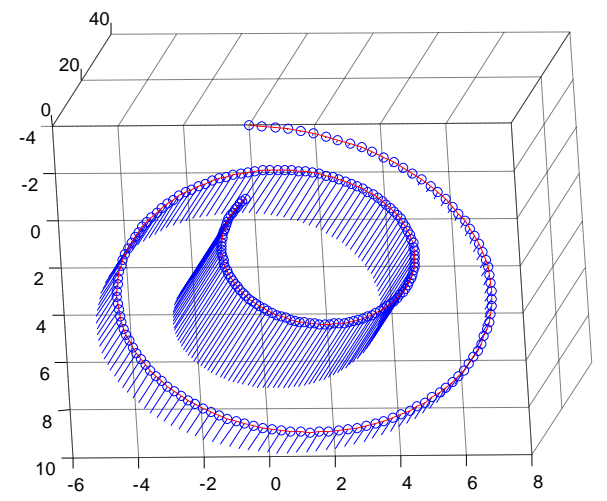

Fig. 4(c) different view of the stem plot $y=4-8 * \exp (-s t / 3)$

Fig. 4(a), Fig. 4(b) and Fig. 4(c) are different views of the function $y=4-8 * \exp (-s t / 3)$.

\section{The Car Number Prediction Model}

The following table gives the data of cars in Forest Lake area Brisbane, during the period of 2000 to 2015 :

TABLE I The Number of Cars in Forest Lake

\begin{tabular}{|c|c|c|c|}
\hline year & $\mathrm{N}$ & year & $\mathrm{N}$ \\
\hline 2001 & 53223 & 2008 & 62291 \\
\hline 2002 & 54982 & 2009 & 62392 \\
\hline 2003 & 55444 & 2010 & 62429 \\
\hline 2004 & 56324 & 2011 & 62553 \\
\hline 2005 & 57003 & 2012 & 62681 \\
\hline 2006 & 58994 & 2013 & 62702 \\
\hline 2007 & 60300 & 2014 & 62806 \\
\hline 2008 & 61993 & 2015 & 62945 \\
\hline 2009 & 62001 & & \\
\hline
\end{tabular}

\section{1) Scatter plot the data:}

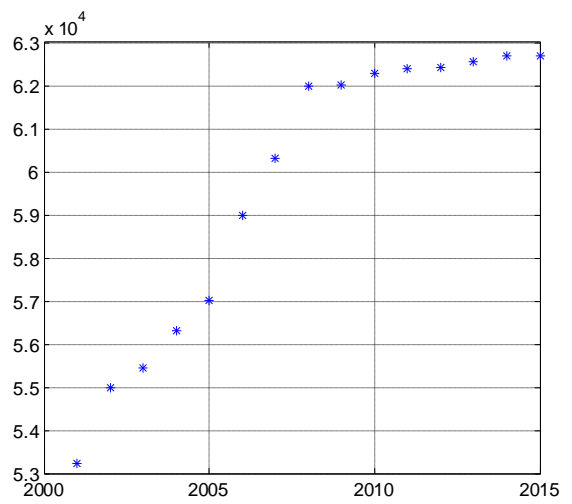

Fig. 5 Scatter points of the number of cars in Forest Lake

\section{2) Fitting the data using formula:}

Using $4^{\text {th }}$ degree ploynomial to fit the data, we get:

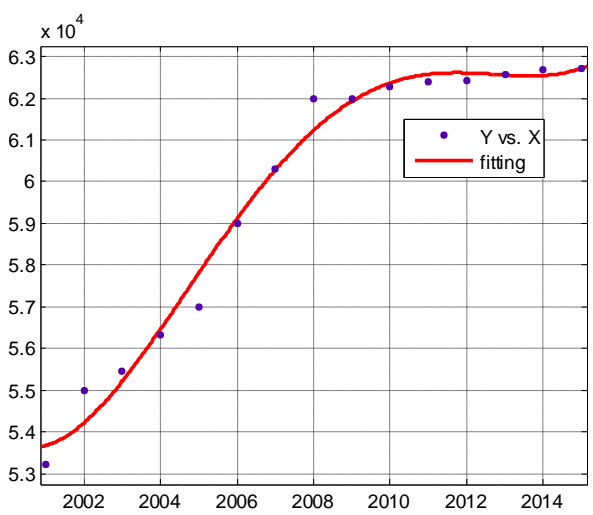

Fig. 6 Fitting the data

The fitting parameters are as follow:

$\mathrm{F}(\mathrm{x})=\mathrm{p} 1^{*} \mathrm{x}^{\wedge} 4+\mathrm{p} 2 * \mathrm{x}^{\wedge} 3+\mathrm{p} 3^{*} \mathrm{x}^{\wedge} 2+\mathrm{p} 4 * \mathrm{x}+\mathrm{p} 5$

where $\mathrm{x}$ is normalized by mean 2008 and std 4.472

Coefficients (with $95 \%$ confidence bounds):

$$
\begin{aligned}
& \text { p1 }= \\
& \text { p2 }=\quad-332.4(51.05,1014) \\
& \text { p3 }=\quad-2540(-722.7,61.48) \\
& \text { p4 }=\quad 3698(2986,4410) \\
& \text { p5 }=6.123 \mathrm{e}+004(6.072 \mathrm{e}+004,6.174 \mathrm{e}+004)
\end{aligned}
$$

The parameters of Goodness of fit are as $\mathrm{f}$ :

SSE: $2.217 \mathrm{e}+006$

R-square: 0.9861

Adjusted R-square: 0.9806

RMSE: 470.9

Fig. 5 is the scatter plot of the number of cars in Forest Lake area Brisbane, and the Fig. 6 is the fitting curve of the scatter points.

\section{Simulation OF Rayleigh Fading}

Rayleigh fading is often experienced in an environment where there are a large number of reflections present. The model of Rayleigh fading uses a statistical approach to analyze the propagation, and can be used in a number of environments. It assumes that the magnitude of a signal varying randomly and fading gradually obey to Rayleigh distribution [7].

Rayleigh distribution is the radial component of the sum of two uncorrelated Gaussian random variables.

Suppose that Z1 and Z2 are independent random variables with standard normal distributions. The magnitude $\mathrm{R}=\mathrm{Z} 21+\mathrm{Z} 22$ of the vector $(\mathrm{Z} 1, \mathrm{Z} 2)$ has the standard Rayleigh distribution [8].

We assume that $\mathrm{X}$ has the Rayleigh distribution with scale parameter $\mathrm{b}$. Then, $\mathrm{X}$ has probability density function (PDF):

$$
f(x)=\frac{x}{b^{2}} \exp \left(-\frac{x^{2}}{2 b^{2}}\right) \text { for } x \in[0, \infty)
$$

$\mathrm{X}$ has cumulative distribution function (CDF): 


$$
F(x)=1-\exp \left(-\frac{x^{2}}{2 b^{2}}\right) \quad \text { for } \quad x \in[0, \infty)
$$

Matlab solution

$\mathrm{N}=10^{\wedge} 6$;

$\mathrm{x}=\operatorname{randn}(1, \mathrm{~N}) ; \%$ Gaussian random samples

$\mathrm{y}=\operatorname{randn}(1, \mathrm{~N}) ; \%$ Gaussian random samples

$\mathrm{z}=\left(\mathrm{x}+\mathrm{j}^{*} \mathrm{y}\right) ; \%$ complex random samples

$\mathrm{zBin}=[0: 0.01: 7]$;

sigma $2=1$

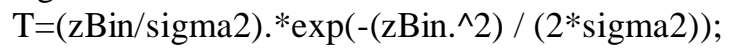

[nzSim zBinSim] $=$ hist $(\operatorname{abs}(\mathrm{z}), \mathrm{zBin})$;

Plot (zBinSim, nzSim/ (N*0.01),'r','LineWidth',1.5);

hold on

plot (zBin, T, 'b-','LineWidth',1.5)

xlabel ('z');

ylabel ('p(z)');

legend ('simulation', 'theory');

title ('PDF of Rayleign');

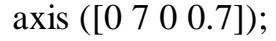

grid on

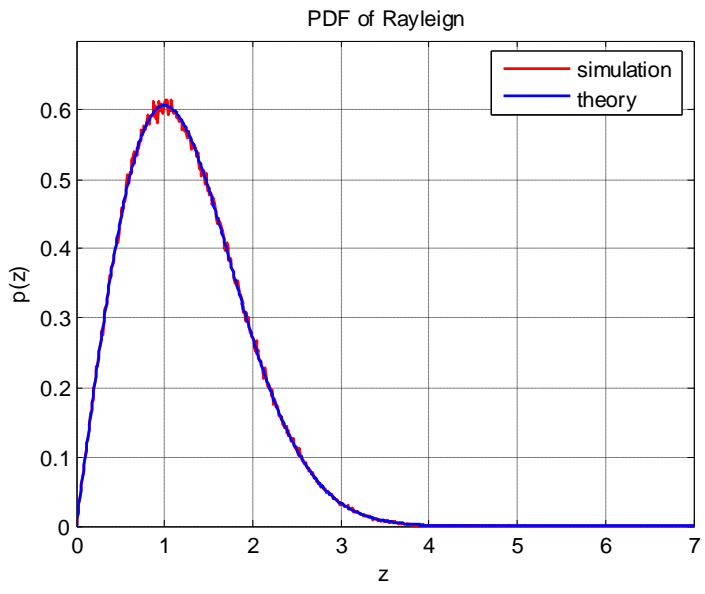

Fig. 7 The Comparison of Simulation and theory

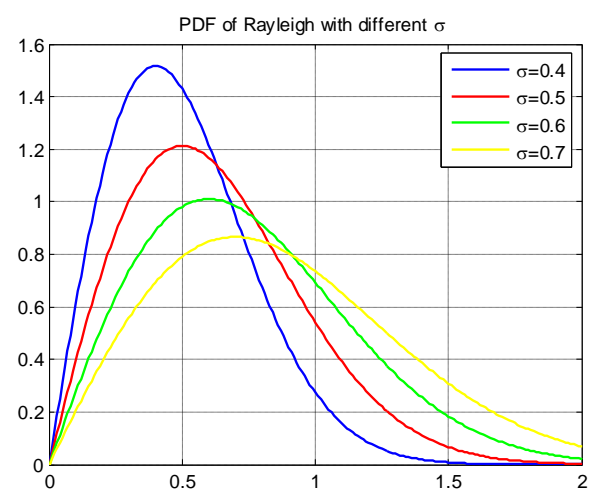

Fig. 8 The PDF of Rayleign Distribution

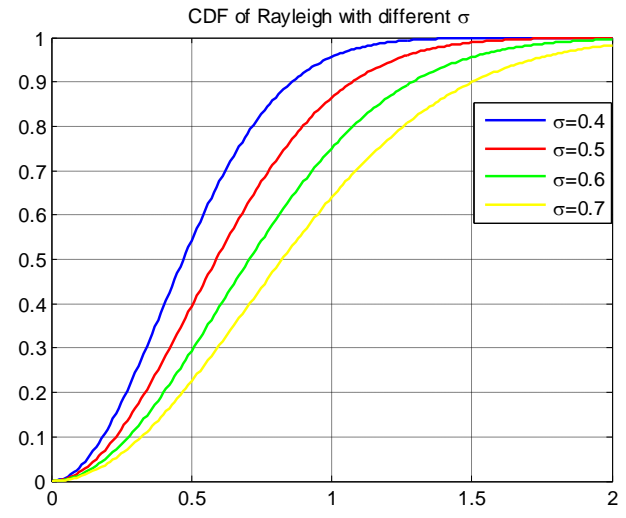

Fig. 9 The CDF of Rayleign Distribution

Using Matlab, we can visually studying the Rayleigh distribution instead of tedious formulas learning. Fig. 7 is the comparison of the simulation data with the theory data. Fig. 8 and Fig. 9 are the PDF and CDF of Rayleigh distribution, respectively.

\section{Conclusion}

Mathematical modelling aims to describe the different aspects of the real world, their interaction, and their dynamics through mathematics. It helps students better understanding many subjects, such as mathematic, physics, signal and systems and so on. Matlab is a wonderful programming software, which is famous for its powerful functions and simple programming languages. It let people free from complicated manual calculation and advanced language program debugging. Using Matlab software in teaching is an advanced method to improve students' academic performance and also a great tool for teacher to do research works.

In this paper, we present the concept and steps of mathematical modeling, and also give some useful examples. It is believed that mathematical modeling is a very using tool in mathematic teaching.

\section{References}

[1] https://en.wikipedia.org/wiki/MATLAB.

[2] "The Mathematical Modeling Cycle", Daniel Maki \& Maynard Thompson; Indiana University.

[3] Carson and C. Cobelli (Eds.), Modelling Methodology for Physiology and Medicine, Academic Press, San Diego, CA, 2001.

[4] Karian, Z.A. and Dudewicz, E.J. (1999), Modern Statistical Systems and GPSS Simulations, 2nd Edition, CRC Press, Florida.

[5] Mann, N.R., Schafer, R. E. and Singpurwalla, N.D. (1974), Methods for Statistical Analysis of Reliability and Life Data, Wiley, New York.

[6] Mudholkar, G.S. and Srivastava, D.K. (1993), " Exponentiated Weibull family for analyzing bathtub failure data", IEEE Transactions on Reliability, vol. 42, 299-302.

[7] Cram er, H. (1951), Mathema tical methods of stat istics (Princeton Unive rsity Press, Princeton) .

[8] Epstein, B., and M. Sobel (1954), Some theorems relevant to life t esting from an expone ntial distribution, Annals of Mathematical Stat istics 25, 373- 381.

[9] Nakagami, Minoru (1940), StllCly on t he r esulta nt 11lnplit.ude of many vibrations whose phases a nd ampli tudes are random, Nippon Elec. Comm. Eng. No. 22, 69- 92. 